\title{
Time-dependent non-Abelian Aharonov-Bohm effect
}

\author{
Max Bright ${ }^{*}$ and Douglas Singleton ${ }^{\dagger}$ \\ Department of Physics, California State University Fresno, Fresno, California 93740-8031, USA \\ (Received 21 January 2015; revised manuscript received 17 March 2015; published 7 April 2015) \\ In this article, we study the time-dependent Aharonov-Bohm effect for non-Abelian gauge fields. We use \\ two well-known time-dependent solutions to the Yang-Mills field equations to investigate the Aharonov- \\ Bohm phase shift. For both of the solutions, we find a cancellation between the phase shift coming from the \\ non-Abelian "magnetic" field and the phase shift coming from the non-Abelian "electric" field, which \\ inevitably arises in time-dependent cases. We compare and contrast this cancellation for the time-dependent \\ non-Abelian case to a similar cancellation which occurs in the time-dependent Abelian case. We postulate \\ that this cancellation occurs generally in time-dependent situations for both Abelian and non-Abelian fields. \\ DOI: 10.1103/PhysRevD.91.085010 \\ PACS numbers: 11.15.-q, 12.38.Aw
}

\section{INTRODUCTION}

The Aharonov-Bohm effect $[1,2]$ is usually investigated in terms of Abelian gauge theories, e.g. electromagnetism formulated via Maxwell's equations. Further, the electromagnetic fields considered in the canonical AharonovBohm effect are static fields. For the vector/magnetic Aharonov-Bohm effect, this means a static vector potential, $\mathbf{A}(\mathbf{r})$, which then translates to a static magnetic field via $\mathbf{B}=\nabla \times \mathbf{A}$. In this article, we wish to consider the Aharonov-Bohm effect in the presence of time-dependent, non-Abelian gauge fields. There has been some prior work on the Aharonov-Bohm effect in the presence of timeindependent, non-Abelian fields [3]. Unlike the Abelian case of electromagnetism, it may not be possible to observe the Aharonov-Bohm effect for static, non-Abelian fields. For the strong interaction, with the non-Abelian $S U(3)$ gauge group, the theory is thought to exhibit confinement. Thus, it is not clear that one could arrange a non-Abelian flux tube that one could control, as is the case with electromagnetism. Further, since the color charges are always confined, one cannot send isolated, unconfined color charges around hypothetical non-Abelian magnetic flux tubes, unlike the Abelian case of electromagnetism, where one can send isolated, unconfined electric charges around Abelian magnetic flux tubes. Despite these experimental obstacles, in this paper, we study the timedependent Aharonov-Bohm effect for non-Abelian fields. The first reason is that the Aharonov-Bohm effect is an important consequence of combining gauge theories with quantum mechanics, and so, it is of interest to see how replacing an Abelian gauge theory by a non-Abelian gauge theory changes (if at all) the Aharonov-Bohm effect. Second, the time-dependent Aharonov-Bohm effect has not been investigated to any great degree, even for Abelian gauge theories. In the two papers $[4,5]$, the time-dependent

\footnotetext{
*neomaxprime@mail.fresnostate.edu

†dougs@csufresno.edu
}

Aharonov-Bohm effect for Abelian fields was investigated and a cancellation was found between the usual magnetic Aharonov-Bohm phase shift and the additional phase shift coming from the electric field, which inevitably occurs for time varying magnetic fields. In this paper, we want to see if a similar cancellation occurs between the non-Abelian magnetic and electric fields.

For our time-dependent non-Abelian field configurations, we take the non-Abelian plane wave solutions of Coleman [6] and the time-dependent Wu-Yang monopole solution [7]. Both solutions satisfy the Yang-Mills field equations for non-Abelian gauge fields of the form

$$
\partial^{\mu} F_{\mu \nu}^{a}+g f^{a b c} A^{\mu b} F_{\mu \nu}^{c}=0,
$$

where $g$ is the coupling constant and $f^{a b c}$ are the group structure constants. $A^{\mu a}$ is the non-Abelian vector potential and the field strength tensor, $F_{\mu \nu}^{a}$ is given by

$$
F_{\mu \nu}^{a}=\partial_{\mu} A_{\nu}^{a}-\partial_{\nu} A_{\mu}^{a}+g f^{a b c} A_{\mu}^{b} A_{\nu}^{c} .
$$

At this point in this paper, we will set $g=1$. For the Coleman non-Abelian plane wave solutions, we find the same cancellation between the non-Abelian magnetic and electric phase shifts that occur in the Abelian case. We also find the same cancellation for the time-dependent Wu-Yang monopole solution. We conclude by giving some remarks as to the similarities between these two time-dependent non-Abelian solutions and the time-dependent Abelian case. We further postulate that the cancellation between the non-Abelian magnetic and electric phase shifts found for the two specific solutions investigated here may be a feature of more general time-dependent non-Abelian solutions.

\section{TIME-DEPENDENT NON-ABELIAN PLANE WAVE SOLUTION}

We begin by reviewing the properties of the two Coleman plane wave solutions. The non-Abelian vector potential for the first $/(+)$ solution is 


$$
A_{\mu}^{(+) a}=\left(x f^{a}\left(\zeta^{+}\right)+y g^{a}\left(\zeta^{+}\right)+h^{a}\left(\zeta^{+}\right), 0,0,0\right),
$$

where $\zeta^{+}=t+z$, in light front coordinates, i.e. $x^{\mu}=$ $\left\{\zeta^{+}, \zeta^{-}, 1,2\right\}$ (the speed of light will be set to unity, $c=1)$. The $(+)$ in the superscript labels this as the light front form of the solution traveling in the negative $z$ direction. The second solution gives waves traveling in the positive $z$ direction. The second solution is only a function of the light front coordinate $\zeta^{-}=t-z$,

$$
A_{\mu}^{(-) a}=\left(0, x f^{a}\left(\zeta^{-}\right)+y g^{a}\left(\zeta^{-}\right)+h^{a}\left(\zeta^{-}\right), 0,0\right) .
$$

Again, the superscript (-) indicates this is the light front form of the solution traveling in the positive $z$ direction. The ansatz functions, $f^{a}\left(\zeta^{ \pm}\right), g^{a}\left(\zeta^{ \pm}\right)$and $h^{a}\left(\zeta^{ \pm}\right)$, are functions of $\zeta^{ \pm}=t \pm z$ but are otherwise arbitrary. First, plugging $A_{\mu}^{(+) a}$ from (3) into (2), the field strength tensor for the light front form of the $(+)$ solution becomes

$$
F_{\mu \nu}^{(+) a}=\left(\begin{array}{cccc}
0 & 0 & -f^{a}\left(\zeta^{+}\right) & -g^{a}\left(\zeta^{+}\right) \\
0 & 0 & 0 & 0 \\
f^{a}\left(\zeta^{+}\right) & 0 & 0 & 0 \\
g^{a}\left(\zeta^{+}\right) & 0 & 0 & 0
\end{array}\right) .
$$

The nonzero components here are $F_{+1}^{(+) a}=-f^{a}\left(\zeta^{+}\right)$and $F_{+2}^{(+) a}=-g^{a}\left(\zeta^{+}\right)$. Next plugging $A_{\mu}^{(-) a}$ from (4) into (2), the field strength tensor for the light front form of the (-) solution becomes

$$
F_{\mu \nu}^{(-) a}=\left(\begin{array}{cccc}
0 & 0 & 0 & 0 \\
0 & 0 & f^{a}\left(\zeta^{-}\right) & g^{a}\left(\zeta^{-}\right) \\
0 & -f^{a}\left(\zeta^{-}\right) & 0 & 0 \\
0 & -g^{a}\left(\zeta^{-}\right) & 0 & 0
\end{array}\right) .
$$

The nonzero components here are $F_{-1}^{(-) a}=-f^{a}\left(\zeta^{-}\right)$and $F_{-2}^{(-) a}=-g^{a}\left(\zeta^{-}\right)$. It is interesting to note that neither of the forms of $F_{\mu \nu}^{a}$ depend on the ansatz function $h^{a}\left(\zeta^{ \pm}\right)$. Also, the non-Abelian term, $f^{a b c} A_{\mu}^{b} A_{\nu}^{c}$, is always zero for both the $(+)$ and the $(-)$ solutions. Thus, the solutions are "very weakly" non-Abelian since this prototypical non-Abelian/ nonlinear term is absent.

Coleman noted that the $(+)$ solution given by (3), in terms of the vector potential, and by (5), in terms of the fields, provides an example of the Wu-Yang ambiguity [8] - that in non-Abelian theories, $F_{\mu \nu}^{a}$ does not contain all the gauge invariant information about a particular solution, as is the case in Abelian gauge theories. Specifically, in terms of the vector potential, one has the quantity

$$
\operatorname{Tr}\left[\mathrm{P} \exp \left(i \oint A_{\mu}^{a} T^{a} d x^{\mu}\right)\right],
$$

where $\mathrm{P}$ indicates path ordering and the $T^{a}$ are the Lie algebra elements. The expression in (7) is identified as the

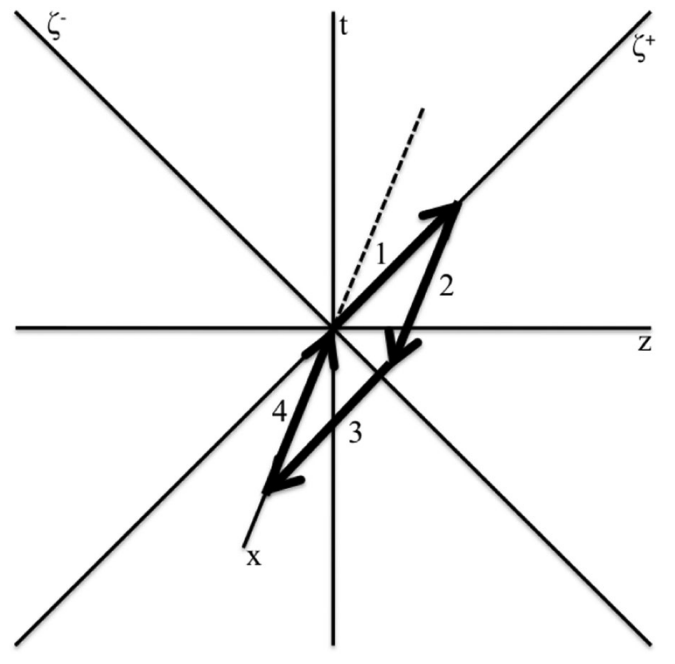

FIG. 1. Unit loop in the $\zeta^{+}-x$ plane.

non-Abelian Aharonov-Bohm phase factor in terms of the potentials. As well, the expression in (7) is the Wilson loop for gauge theories [9].

We now consider a unit loop in the $\zeta^{+}-x^{1}$ plane (i.e. the $\zeta^{+}-x$ plane) and starting from $x=0$ and $\zeta^{+}=0$ and going in the direction given in Fig. 1. For this loop the integral in the exponent in (7) becomes

$$
\begin{aligned}
\oint A_{\mu}^{(+) a} d x^{\mu}= & \int_{1} A_{+}^{(+) a} d \zeta^{+}+\int_{2} A_{x}^{(+) a} d x-\int_{3} A_{+}^{(+) a} d \zeta^{+} \\
& -\int_{4} A_{x}^{(+) a} d x \\
= & \int_{1} h^{a}\left(\zeta^{+}\right) d \zeta^{+}-\int_{3}\left[f^{a}\left(\zeta^{+}\right)+h^{a}\left(\zeta^{+}\right)\right] d \zeta^{+} .
\end{aligned}
$$

The second and fourth integrals [i.e. $\int_{2} A_{x}^{(+) a} d x$ and $\left.\int_{4} A_{x}^{(+) a} d x\right]$ are zero since $A_{x}^{(+) a}=0$ for the $(+)$ solution in (3). For the third integral, we get $-\int_{3}\left[f^{a}\left(\zeta^{+}\right)+\right.$ $\left.h^{a}\left(\zeta^{+}\right)\right] d \zeta^{+}$, since for this leg, $x=1$ and $y=0$ and one is going backward along the $\zeta^{+}$direction, while for the first integral, we get $\int_{1} h^{a}\left(\zeta^{+}\right) d \zeta^{+}$since $x=y=0$. Note that $\int_{1} h^{a}\left(\zeta^{+}\right) d \zeta^{+}$and $-\int_{3} h^{a}\left(\zeta^{+}\right) d \zeta^{+}$do not cancel, due to the path ordering in (7). Taking the path ordering into account and combining (8) and (7) one gets

$$
\begin{aligned}
\operatorname{Tr} & {\left[\exp \left(i T^{a} \int_{1} h^{a}\left(\zeta^{+}\right) d \zeta^{+}\right)\right.} \\
& \left.\times \exp \left(-i T^{b} \int_{3}\left[f^{b}\left(\zeta^{+}\right)+h^{b}\left(\zeta^{+}\right)\right] d \zeta^{+}\right)\right] .
\end{aligned}
$$

From (9) it is evident that there is no cancellation of the $h^{a}$ functions due to the nontrivial commutation relationship of 
$T^{a}$ and $T^{b}$. The above result is equivalent to the result given in Eq. (8) of [6].

Now, the field strength version of (7) (and the field strength version of the Aharonov-Bohm phase for nonAbelian theories [10]) is

$$
\operatorname{Tr}\left[\mathcal{P} \exp \left(\frac{i}{2} \int F_{\mu \nu}^{a} T^{a} d \sigma^{\mu \nu}\right)\right],
$$

where $d \sigma^{\mu \nu}$ is the area and $\mathcal{P}$ means "area" ordering [11]. For the unit area spanning the unit loop in the $\zeta^{+}-x$ plane, the differential area is given by $d \sigma^{+1}=d \zeta^{-} d x=$ $(d t-d z) d x$. The reason that $d \sigma^{+1}$ has $d \zeta^{-}$rather than $d \zeta^{+}$ is that the area vector should be perpendicular to the surface spanned by $\zeta^{+}-x$, and it is $\zeta^{-}$, not $\zeta^{+}$, which is perpendicular to $d \sigma^{+1}$, as seen in Fig. 1. Similarly, $\zeta^{+}$is perpendicular to $d \sigma^{-1}$. Note that for this unit square the area ordering denoted by $\mathcal{P}$ is simple since there is only one area vector. With only a single area vector the issue of ordering does not arise. As a result of the above discussion, the integral in the exponential in (10) for the unit loop in the $\zeta^{+}-x^{1}$ plane becomes

$$
\int F_{+1}^{(+) a} T^{a} d \sigma^{+1}=-T^{a} \int\left(f^{a}(t+z)\right)(d t d x-d z d x)=0 .
$$

This integral is zero since $\int f^{a}(t+z) d t=\int f^{a}(t+z) d z$, which is due to the $\zeta^{+}$functional dependence of $f^{a}$, but there is a sign difference between the $d t$ integration and the $d z$ integration. For a unit loop in the $\zeta^{+}-x^{2}$ plane (i.e. the $\zeta^{+}-y$ plane), we would find a similar result as in (11), except for the replacement $F_{+1}^{(+) a} \rightarrow F_{+2}^{(+) a}=-g^{a}(t+z)$. Since the area for this loop is $d \sigma^{+2}=d \zeta^{-} d y=(d t-d z) d y$, we again get $\int g^{a}(t+z) d t=\int g^{a}(t+z) d z$, which then cancels because of the $d \zeta^{-}$in the unit area element. The same result also holds for the (-) solution from (6). In this case, the unit loop is in the $\zeta^{-}-x^{1}$ plane (i.e. the $\zeta^{-}-x$ plane) or the $\zeta^{-}-x^{2}$ plane (i.e. the $\zeta^{-}-y$ plane). The perpendicular areas in this case will be $d \sigma^{-1}=d \zeta^{+} d x=(d t+d z) d x$ and $d \sigma^{-2}=d \zeta^{+} d y=(d t+d z) d y$. Now, the relevant integrals will be $\int f^{a}(t-z) d t=-\int f^{a}(t-z) d z$ and $\int g^{a}(t-z) d t=-\int g^{a}(t-z) d z$, so that one again gets zero for the area integrals like $\int f^{a}(t-z) T^{a}(d t d x+$ $d z d x)=0$ and $\int g^{a}(t-z) T^{a}(d t d y+d z d y)=0$. This vanishing of the area integral of the non-Abelian field strengths, $F_{\mu \nu}^{( \pm) a}$, occurs for both of the time-dependent, non-Abelian solutions we examined. Although we do not have a general proof, we conjecture that this cancellation will occur generally for time-dependent, non-Abelian solutions. An important point to note is that it is only the time-dependent part of $\frac{1}{2} \int F_{\mu \nu}^{a} T^{a} d \sigma^{\mu \nu}$ which is conjectured to vanish-the static parts of the fields still give the usual non-Abelian Aharonov-Bohm phase.

In the light front coordinates, the split between the nonAbelian electric and magnetic field components is not so straightforward. Just as the $\zeta^{ \pm}$coordinates are mixtures of space and time coordinates, so too $F_{ \pm \mu}^{a}$ are mixtures of nonAbelian electric and magnetic field components. For this reason, we now perform the above analysis of $\oint A_{\mu}^{a} T^{a} d x^{\mu}$ and $\frac{1}{2} \int F_{\mu \nu}^{a} T^{a} d \sigma^{\mu \nu}$ in Cartesian coordinates $(t, x, y, z)$. We do this for the $(+)$ solution of (3) but the same analysis applies to the (-) solution of (4). The $x, y$ components of the $(+)$ solution are the same in light front and Cartesian coordinates. The time and $z$ components are obtained via $A_{0}^{(+) a}=\frac{1}{2}\left(A_{+}^{(+) a}+A_{-}^{(+) a}\right)$ and $A_{z}^{(+) a}=\frac{1}{2}\left(A_{+}^{(+) a}-A_{-}^{(+) a}\right)$, which is the same way one transforms between $\zeta_{ \pm}$and $t, z$. Also, note that the superscript $(+)$ labels the solution while the subscripts label the \pm components of this solution. Thus, in Cartesian coordinates, the $(+)$ solution is

$$
\begin{aligned}
A_{\mu}^{(+) a}= & \frac{1}{2}\left(x f^{a}\left(\zeta^{+}\right)+y g^{a}\left(\zeta^{+}\right)+h^{a}\left(\zeta^{+}\right), 0,0, x f^{a}\left(\zeta^{+}\right)\right. \\
& \left.+y g^{a}\left(\zeta^{+}\right)+h^{a}\left(\zeta^{+}\right)\right),
\end{aligned}
$$

where now $\mu=(0,1,2,3)$ rather than $\mu=(+,-, 1,2)$. The Cartesian field strength tensor following from (12) is

$$
F_{\mu \nu}^{(+) a}=\frac{1}{2}\left(\begin{array}{cccc}
0 & -f^{a}\left(\zeta^{+}\right) & -g^{a}\left(\zeta^{+}\right) & 0 \\
f^{a}\left(\zeta^{+}\right) & 0 & 0 & f^{a}\left(\zeta^{+}\right) \\
g^{a}\left(\zeta^{+}\right) & 0 & 0 & g^{a}\left(\zeta^{+}\right) \\
0 & -f^{a}\left(\zeta^{+}\right) & -g^{a}\left(\zeta^{+}\right) & 0
\end{array}\right),
$$

where again the indices $\mu, \nu$ are given by $\mu, \nu=(0,1,2,3)$ rather than $\mu, \nu=(+,-, 1,2)$. As a check, it is easy to verify that (12) and (13) satisfy the Yang-Mills field equations (1). In the form (13), the split between non-Abelian electric and magnetic components is obvious - the first row and column are the electric components and the $3 \times 3$ submatrix below and to the right of the first row and the first column are the magnetic components.

Thus, in Cartesian coordinates, the loop integral in the exponent in (7) becomes

$$
\begin{aligned}
\oint T^{a} A_{\mu}^{(+) a} d x^{\mu}= & \frac{1}{2}\left(\int_{1} T^{a} A_{0}^{(+) a} d t+\int_{1} T^{a} A_{z}^{(+) a} d z\right) \\
& -\frac{1}{2}\left(\int_{3} T^{b} A_{0}^{(+) b} d t+\int_{3} T^{b} A_{z}^{(+) b} d z\right) \\
= & \int_{1} T^{a} h^{a}\left(\zeta^{+}\right)(d t \text { or } d z) \\
& -\int_{3} T^{b}\left[f^{b}\left(\zeta^{+}\right)+h^{b}\left(\zeta^{+}\right)\right](d t \text { or } d z) .
\end{aligned}
$$

Again, paths 2 and 4 do not contribute since the $x$ component of the $(+)$ solution in Cartesian coordinates 
is zero, $A_{x}^{(+) a}=0$, as was the case for the solution in light front coordinates. Paths 1 and 3 have equal components in the $t$ and $z$ directions and thus, pick out the gauge field components $A_{0}^{(+) a}$ and $A_{3}^{(+) a}$. Along path $1, x=y=0$, so only $h^{a}$ appears; while for path $3, x=1$ and $y=0$, so now both $h^{a}$ and $f^{a}$ appear. Because of the dependence of $h^{a}, f^{a}$ on $\zeta^{+}=t+z$, the $d t$ and $d z$ integrals of these functions are the same. The end result is that the loop integral of the gauge field gives the same results in light front coordinates, (8), and in Cartesian coordinates, (14). Thus the phase shift calculated by the loop integral of the gauge fields-Eq. (9) - is the same in both Cartesian and light front coordinates.

Next, we calculate the surface area integral, $\frac{1}{2} \int F_{\mu \nu}^{a} T^{a} d \sigma^{\mu \nu}$, for the unit area from Fig. 1. Taking into account the antisymmetry of $F_{\mu \nu}^{a}$ and $d \sigma^{\mu \nu}$ under exchange of the indices, the $(+)$ solution, in Cartesian coordinates, and with the surface in the $\zeta^{+}-x$ plane from Fig. 1, we find that the surface area integral of the field strength tensor is

$$
\begin{aligned}
\frac{1}{2} \int & F_{\mu \nu}^{(+) a} T^{a} d \sigma^{\mu \nu} \\
& =T^{a}\left(\int F_{01}^{(+) a} d x^{0} d x^{1}+\int F_{13}^{(+) a} d x^{1} d x^{3}\right) \\
& =T^{a}\left(\int\left(-f^{a}\left(\zeta^{+}\right)\right) d t d x+\int f^{a}\left(\zeta^{+}\right) d x d z\right) .
\end{aligned}
$$

There are two integrals- $\int F_{02}^{(+) a} d x^{0} d x^{2}$ and $\int F_{23}^{(+) a} d x^{2} d x^{3}-$ which are absent from (15) since for the unit area in the $\zeta^{+}-x$ plane, $d x^{2}=d y=0$. If we take a surface in the $\zeta^{+}-y$ plane, then these two integrals would appear and instead, $\int F_{01}^{(+) a} d x^{0} d x^{1}$ and $\int F_{13}^{(+) a} d x^{1} d x^{3}$ from (15) would vanish. The two integrals in (15) cancel since $\int f^{a}(t+z) d t=$ $\int f^{a}(t+z) d z$, due to the fact that $f^{a}$ depends on $\zeta^{+}=t+z$. Similarly, the two integrals for the $\zeta^{+}-y$ plane cancel since $\int g^{a}(t+z) d t=\int g^{a}(t+z) d z$, due to the fact that $g^{a}$ depends on $\zeta^{+}=t+z$. Thus, in both the light front and the Cartesian form, the $(+)$ solution has $\frac{1}{2} \int F_{\mu \nu}^{(+) a} T^{a} d \sigma^{\mu \nu}=0$. In (15), it is more apparent that the cancellation is between the non-Abelian electric pieces (the $F_{0 i}^{a}$ integrals) and the corresponding non-Abelian magnetic pieces (the $F_{i j}^{a}$ integrals).

As Coleman already noted, the expression $\oint A_{\mu}^{a} T^{a} d x^{\mu}$ in (8) does not in general agree with $\int F_{\mu \nu}^{a} T^{a} d \sigma^{\mu \nu}$ in (11) for the light front solutions in (3) and (4). We have shown that the same is true (as it should be) in the Cartesian coordinate version of the solution. This is taken by Coleman [6] as an illustration of the Wu-Yang ambiguity [8] - in non-Abelian gauge theories, not all the gauge invariant information is contained in the field strength tensor.

\section{TIME-DEPENDENT NON-ABELIAN WU-YANG MONOPOLE SOLUTION}

We now examine the issue of the time-dependent, nonAbelian Aharonov-Bohm phase for the time-dependent $S U$ (2) Wu-Yang monopole solution, where $f^{a b c} \rightarrow \epsilon^{a b c}$. This solution is different in two respects from the nonAbelian plane waves of the previous section. First, in contrast to the non-Abelian plane wave solution of the previous section, the time-dependent $S U(2)$ Wu-Yang monopole solution is more strongly non-Abelian since the prototypical non-Abelian term $\left(\epsilon^{a b c} A_{\mu}^{b} A_{\nu}^{c}\right)$ is nonzero. Second, in the light front coordinates of the previous section, the non-Abelian electric and magnetic components of the field strength tensor are mixed up (combined) in nonzero components like $F_{+1}^{(+) a}$ or $F_{+2}^{(+) a}$. For the timedependent $S U(2)$ Wu-Yang monopole solution of this section, the field strength tensor is split into separate non-Abelian electric (e.g. $E_{x}^{a}=F_{01}^{a}$ ) and magnetic (e.g. $B_{z}^{a}=-F_{12}^{a}$ ) pieces. The vector potential for the timedependent Wu-Yang solution is given by [7]

$$
A_{0}^{a}=0 ; \quad A_{i}^{a}=-\epsilon_{a i j} \frac{x^{j}}{r^{2}}[1+f(r, t)],
$$

where $f(r, t)$ is a radial and time-dependent function and $\epsilon_{i j k}$ is the $S U(2)$ Levi-Civita structure constant of the group. For this time-dependent solution, one works specifically with the $S U(2)$ group, whereas for the nonAbelian plane wave solution of the last section, the Lie group was arbitrary. As pointed out in the previous section, the non-Abelian part of the field strength tensor did not play a big role in the Coleman solutions. For this reason, the exact nature of the non-Abelian group was not so crucial for the Coleman plane wave solutions. From (16), one can immediately find the associated field strength tensor as

$$
\begin{aligned}
F_{0 i}^{a}= & -\epsilon_{a i j} \frac{x^{j}}{r^{2}} \dot{f}(r, t) \\
F_{i j}^{a}= & -\left(\frac{1+f}{r^{2}}\right)^{\prime} \frac{x^{i} x^{k} \epsilon_{a j k}-x^{j} x^{k} \epsilon_{a i k}}{r}-2 \frac{1+f}{r^{2}} \epsilon_{a j i} \\
& -(1+f)^{2} \frac{x^{a} x^{k}}{r^{4}} \epsilon_{i j k},
\end{aligned}
$$

where the dot denotes a time derivative and the prime denotes a radial derivative. The first two terms in $F_{i j}^{a}$ are the Abelian/pure curl part of the non-Abelian magnetic field (i.e. $\partial_{i} A_{j}^{a}-\partial_{j} A_{i}^{a}=\left[\nabla \times \mathbf{A}^{a}\right]_{k}$ ). Also the non-Abelian electric field, $E_{i}^{a}=F_{0 i}^{a}=-\partial_{t} A_{i}^{a}$, is Abelian in character since the prototypical, non-Abelian piece, $\epsilon^{a b c} A_{0}^{b} A_{i}^{c}$, is not present. One can thus show, generally, that there is a cancellation between the Abelian part of the non-Abelian electric field and the Abelian part of the non-Abelian magnetic field, $\left[\nabla \times \mathbf{A}^{a}\right]_{i}$. For this Abelian part of the fields 
from (17), the area integral that appears in the field strength version of the Aharonov-Bohm phase is

$$
\begin{aligned}
& \int \mathbf{E}^{a} \cdot d \mathbf{x} d t+\int \mathbf{B}_{(\text {Abelian })}^{a} \cdot d \mathbf{a} \\
& =-\int \partial_{t} \mathbf{A}^{a} \cdot d \mathbf{x} d t+\int \nabla \times \mathbf{A}^{a} \cdot d \mathbf{a} \\
& =-\oint \mathbf{A}^{a} \cdot d \mathbf{x}+\oint \mathbf{A}^{a} \cdot d \mathbf{x}=0,
\end{aligned}
$$

where for the first, "electric area" integral, we have done the time integration to get $-\oint \mathbf{A}^{a} \cdot d \mathbf{x}$; for the second, magnetic area integral, we have used Stokes's theorem to get $\oint \mathbf{A}^{a} \cdot d \mathbf{x}$, which then cancels the first, electric term. Note, for the first, electric term, one leg of the area is a time piece.

However, the last, non-Abelian term in the magnetic field-i.e. the term $\epsilon_{i j k} \epsilon^{a b c} A_{i}^{b} A_{j}^{c}=-(1+f)^{2} \frac{x^{a} x^{k}}{r^{4}} \epsilon_{i j k}$ in (17) - could give a nonzero contribution to the calculation, so at first sight one would not, in general, expect the same kind of cancellation between the non-Abelian electric and magnetic parts that occurred in (11) for the Coleman solution. To this end, we will look at the full non-Abelian fields of (17) for a specific contour bounding a specific area, and we will see that there is still a complete cancellation between the electric and magnetic parts, as there was for the Coleman solutions of the previous section. The specific contour we take is a hoop of radius $R$ in the $x-y$ plane going in the counterclockwise direction. The area spanning this contour is a circle with area $\pi R^{2} \hat{\mathbf{z}}$, i.e. pointing in the $+\hat{\mathbf{z}}$ direction. The surface and contour are shown in Fig. 2. In evaluating the electric and magnetic parts of (17), we will consider infinitesimal path lengths, $R \Delta \varphi$, and infinitesimal areas, $R^{2} \Delta \varphi / 2$ see Fig. 3.

We begin by looking at the non-Abelian electric contribution $\int F_{0 i}^{a} d x^{0} d x^{i}=\int \mathbf{E}^{a} \cdot d \mathbf{x} d t$. For the $F_{0 i}^{a}$ from (17), one finds

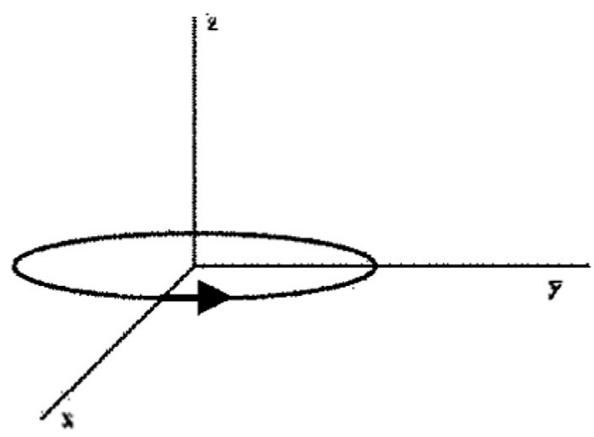

FIG. 2. Hoop of radius $R$ in the $x-y$ plane.

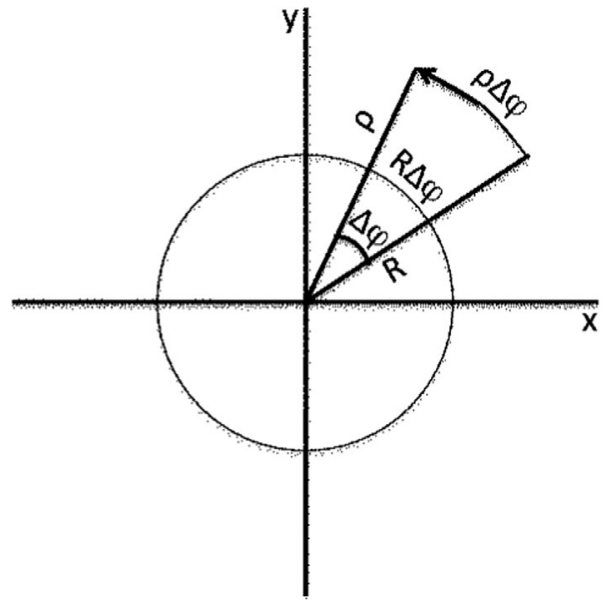

FIG. 3. Infinitesimal path and area for the canonical AharonovBohm setup.

$$
\begin{aligned}
\int F_{0 i}^{a} d x^{0} d x^{i} & =-\epsilon_{a i j} \int \frac{\dot{f}(R, t)}{R^{2}} x^{j} d t d x^{i} \\
& =\frac{1}{R^{2}} \int[\mathbf{x} \times d \mathbf{x}]^{a} \int \dot{f}(R, t) d t \\
& =\Delta \varphi \Delta f \delta^{a 3},
\end{aligned}
$$

where we have used the fact that $\int[\mathbf{x} \times d \mathbf{x}]^{a}=\Delta \varphi R^{2} \delta^{a 3}$, i.e. this integral gives twice the area of an infinitesimal wedge from the surface in Fig. 2 (see also Fig. 3). The direction of the area is in the 3 or $+\hat{\mathbf{z}}$ direction. From the $\epsilon$ symbol in (19), with $i, j=1,2$, the color index is fixed as $a=3$. To make $a=3$ explicit, we have inserted $\delta^{a 3}$ into the final expression in (19). The time integration has been done for an infinitesimal interval $\Delta t$, so $\dot{f}(R, t) \Delta t=\Delta f$.

Now we calculate the non-Abelian magnetic contribution to the phase. We do this in two separate pieces: the Abelian part, $\partial_{i} A_{j}^{a}-\partial_{j} A_{i}^{a}$, and the non-Abelian part, $\epsilon_{i j k} \epsilon^{a b c} A_{i}^{b} A_{j}^{c}$. First, for the Abelian part, we have

$$
\begin{aligned}
B_{k(\text { Abelian })}^{a} & =-\partial_{i} A_{j}^{a}+\partial_{j} A_{i}^{a} \\
& =\left(\frac{1+f}{r^{2}}\right)^{\prime} \frac{x^{i} x^{k} \epsilon_{a j k}-x^{j} x^{k} \epsilon_{a i k}}{r}+2 \frac{1+f}{r^{2}} \epsilon_{a j i} .
\end{aligned}
$$

For our contour from Fig. 2, the first term in (20) has $i, j=1,2$ since we are in the $x-y$ plane. But as well, for the summation over the $k$ index, we also need $k=1$ or $k=2$. For $k=3$, we would have $x^{3}=z$, but $z=0$ for the contour and surface we are using, so for $k=3$, the first term in (20) is zero. Taking all this into account, if we look at $i=1$ and $j=2$, we find that the indexed part of the first term in (20) is

$$
x^{1} x^{1} \epsilon_{a 21}-x^{2} x^{2} \epsilon_{a 12}=\left(-x^{2}-y^{2}\right) \delta^{a 3}=-r^{2} \delta^{a 3},
$$


since in the $x$ - $y$ plane $x^{2}+y^{2}=r^{2}$. Note also that the color index, $a$, is forced to be $a=3$. With this, the first term of (20) becomes

$$
\begin{aligned}
& -\delta^{a 3} \int\left(\frac{1+f}{r^{2}}\right)^{\prime} r^{2} d r d \varphi \\
& =-\delta^{a 3} \int f^{\prime} d r d \varphi+2 \delta^{a 3} \int \frac{(1+f)}{r} d r d \varphi .
\end{aligned}
$$

Next, since our surface and contour from Fig. 2 are in the $x-y$ plane, this means $i, j=1,2$, thus, for the last term in (20), this implies that $a=3$, and we find that this term becomes

$2 \int \frac{(1+f)}{r^{2}} \epsilon_{321} d x^{1} d x^{2}=-2 \delta^{a 3} \int \frac{(1+f)}{r} d r d \varphi$,

where in the $x-y$ plane $d x^{1} d x^{2}$ become $r d r d \varphi$.

The second term in (21) will cancel the term in (22). The first term in (21) will have a $\Delta \varphi$ from the infinitesimal $\varphi$ integration. Then using infinitesimal notation for the $r$ integration (i.e. $\frac{\Delta f}{\Delta r} \Delta r=\Delta f$ ), we arrive at

$$
\int \mathbf{B}_{(\text {Abelian })}^{a} \cdot d \mathbf{a}=-\Delta \varphi \Delta f \delta^{a 3}
$$

which then cancels the electric contribution (19). Thus, at this point we have confirmed, with specific contours and areas, the cancellation between the electric and "Abelian magnetic" parts of the non-Abelian Aharonov-Bohm phase, which was shown generally in (18).

The final piece we need to deal with is the prototypical non-Abelian piece of the magnetic contribution, namely

$$
\int \epsilon_{i j k} \epsilon^{a b c} A_{i}^{b} A_{j}^{c} d x^{i} d x^{j}=-\int(1+f)^{2} \frac{x^{a} x^{k}}{r^{4}} \epsilon_{i j k} d x^{i} d x^{j}=0 .
$$

This piece is seen to vanish since $i, j=1,2$ due to the contour/area from Fig. 2 lying in the $x-y$ plane. This forces $k=3$ so that $x^{k} \rightarrow z$, but since we are in the $x-y$ plane $z=0$, so this prototypical non-Abelian contribution vanishes. Thus, as for the Coleman plane wave solution of the previous section, we find a cancellation between the electric and magnetic parts of the non-Abelian AharonovBohm phase. Although here we have shown this cancellation for only two types of time-dependent non-Abelian solutions and with specific contours, we nevertheless advance the hypothesis that this cancellation is a general feature of both Abelian and non-Abelian Aharonov-Bohm phases for time-dependent fields.

We conclude this section by noting that, like the Coleman solutions, the time-dependent Wu-Yang monopole solution shows a nonzero phase when calculated using the potential

$$
\oint A_{\mu}^{a} d x^{\mu} \rightarrow-\oint \mathbf{A} \cdot d \mathbf{x} \rightarrow-\Delta A_{i}^{a} \Delta x^{i},
$$

where in the last step we are considering an infinitesimal path length as in Fig. 3, in conjunction with an infinitesimal change in the potential $\Delta A_{i}^{a}$. Using the form for $A_{i}^{a}$ from (16), we find

$$
\begin{aligned}
-\Delta A_{i}^{a} \Delta x^{i} & =-\Delta(1+f(R, t))\left(-\frac{1}{R^{2}} \epsilon_{a i j} x^{j} \Delta x^{i}\right) \\
& \rightarrow-(\Delta f)\left(\frac{1}{R^{2}}[\mathbf{x} \times \Delta \mathbf{x}]^{a}\right) .
\end{aligned}
$$

In the last step, we have canceled two minus signs but have switched the $i$ and $j$ index which then gives an additional minus sign. Also, we have used $\Delta f=$ $\partial_{t} f(R, t) \Delta t=\frac{\Delta f}{\Delta t} \Delta t$. Finally, we again use $[\mathbf{x} \times \Delta \mathbf{x}]^{a}=$ $\Delta \varphi R^{2} \delta^{a 3}$ and find that

$\oint A_{\mu}^{a} d x^{\mu} \rightarrow-\oint \mathbf{A} \cdot d \mathbf{x} \rightarrow-\Delta A_{i}^{a} \Delta x^{i} \rightarrow-\delta^{a 3} \Delta \varphi \Delta f$.

This produces only the non-Abelian magnetic phase contribution

from the fields calculation. In the next section, we will discuss this nonequivalence between the time-dependent Aharonov-Bohm phase shift, calculated using the potentials versus the field strengths, by comparing it with the time-dependent Abelian Aharonov-Bohm phase case.

\section{COMPARISON WITH TIME-DEPENDENT ABELIAN AHARONOV-BOHM EFFECT}

In the previous two sections, we discussed the timedependent Aharonov-Bohm phase shift for non-Abelian fields for two specific solutions (the Coleman plane wave solutions and the time-dependent $\mathrm{Wu}$-Yang monopole) and two specific contours (a unit square in the $\zeta^{+}-x$ plane and a ring of radius $R$ in the $x-y$ plane). For the Coleman solutions, the field strength tensors had no contribution from the non-Abelian term, $f^{a b c} A_{\mu}^{b} A_{\nu}^{c}$, and the functional form of the field strengths was essentially the same as for the Abelian plane wave solutions. One distinction between the Coleman non-Abelian plane waves and the Abelian planes waves is that superposition does not apply for the Coleman plane waves. For the time-dependent Wu-Yang monopole solution, the electric field strength terms had no contribution from the non-Abelian part of the solution, but the magnetic field did. However, this non-Abelian part for the magnetic field was found not to contribute to the Aharonov-Bohm phase for the specific paths and surfaces we used, which are shown in Fig. 2 (see also Fig. 3). We now review the Abelian, time-dependent Aharonov-Bohm effect and draw parallels with the non-Abelian case.

The time-independent Abelian Aharonov-Bohm effect has been well studied theoretically and also been confirmed 
experimentally [12,13] (see [14] for a recent experimental tests using tunneling). In contrast, the timedependent Abelian Aharonov-Bohm effect has received much less attention. Some of the papers dealing with the time-dependent Abelian Aharonov-Bohm effect are $[4,5,15,16]$. Paper [15] predicts a time-shifting interference pattern for the time-dependent Abelian Aharonov-Bohm effect, while [4,5] find a cancellation of the time-dependent electric and magnetic contributions and thus a nonshifting interference pattern. The few experiments done on the timedependent Abelian Aharonov-Bohm effect (see $[17,18]$ and also [19] for an "accidental" test of the time-dependent Abelian Aharonov-Bohm effect) confirm the predictions of $[4,5]$, but each experiment had problems. Thus, a definitive test of the time-dependent Abelian Aharonov-Bohm effect, to distinguish between the predictions of [15] and [4,5], still needs to be done.

The vector potential for a time-dependent AharonovBohm solenoid is given by [4,5] (we use cylindrical coordinates $\rho, \varphi$ and the magnetic flux tube has a radius $R$ )

$$
\begin{array}{cl}
\mathbf{A}_{\text {in }}=\frac{\rho B(t)}{2} \hat{\boldsymbol{\varphi}} & \text { for } \rho<\mathrm{R} \\
\mathbf{A}_{\text {out }}=\frac{B(t) R^{2}}{2 \rho} \hat{\boldsymbol{\varphi}} & \text { for } \rho \geq \mathrm{R} .
\end{array}
$$

To begin with, we have taken the scalar potential, $\phi$, as zero. We return to this point later since there are nonsingle valued gauges where there is a nonzero and a nonsingle valued $\phi$. The possibility of a nonsingle valued $\phi$ leads to something similar to the Wu-Yang ambiguity but for timedependent Abelian fields. The magnetic and electric fields coming from (28) are

$$
\begin{aligned}
\mathbf{B}_{\text {in }} & =\nabla \times \mathbf{A}_{\text {in }}=B(t) \hat{z} \quad \text { for } \rho<\mathrm{R} \\
\mathbf{B}_{\text {out }} & =\nabla \times \mathbf{A}_{\text {out }}=0 \quad \text { for } \rho \geq \mathrm{R},
\end{aligned}
$$

and

$$
\begin{gathered}
\mathbf{E}_{\text {in }}=-\frac{\partial \mathbf{A}_{\text {in }}}{\partial t}=-\frac{\rho \dot{B}(t)}{2} \hat{\boldsymbol{\varphi}} \quad \text { for } \rho<\mathrm{R} \\
\mathbf{E}_{\text {out }}=-\frac{\partial \mathbf{A}_{\text {out }}}{\partial t}=-\frac{\dot{B}(t) R^{2}}{2 \rho} \hat{\boldsymbol{\varphi}} \quad \text { for } \rho \geq \mathrm{R} .
\end{gathered}
$$

Evaluating the Aharonov-Bohm phase using fields (29) and (30), for the infinitesimal path and associated area in Fig. 3, gives

$$
\int \mathbf{E}_{\text {out }} \cdot d \mathbf{x} d t+\int \mathbf{B}_{\text {in }} \cdot d \mathbf{a} \rightarrow\left(\mathbf{E}_{\text {out }} \cdot \Delta \mathbf{x} \Delta t\right)+\left(\mathbf{B}_{\text {in }} \cdot \Delta \mathbf{a}\right) .
$$

By expanding $\mathbf{B}_{\text {in }}=\mathbf{B}_{0}+\dot{\mathbf{B}} \Delta t$ and identifying the infinitesimal path, $\Delta \mathbf{x}=\rho \Delta \varphi \hat{\boldsymbol{\varphi}}$, and the area, $\Delta \mathbf{a}=\frac{1}{2} R^{2} \Delta \varphi \hat{\mathbf{z}}$, Eq. (31) becomes
$-\left(\frac{\dot{B} R^{2}}{2} \Delta \varphi \Delta t\right)+\left(\frac{B_{0} R^{2} \Delta \varphi}{2}+\frac{\dot{B} R^{2}}{2} \Delta \varphi \Delta t\right)=\frac{B_{0} R^{2} \Delta \varphi}{2}$.

The time-dependent parts of the phase shift cancel each other, while the static Aharonov-Bohm phase shift, due to $B_{0}$, remains.

Strictly, in order to extend the above analysis for an infinitesimal interval $\Delta t$ to an arbitrary time interval, one would need to consider a linearly increasing magnetic flux, $\mathbf{B}_{\text {in }}=\mathbf{B}_{0}+\mathbf{B}_{1} t$, where $\mathbf{B}_{0}$ and $\mathbf{B}_{1}$ are constant. For such a linearly changing magnetic field, all of Maxwell's equations are exactly satisfied with the fields given by (29) and (30). However, for other time dependencies, the radial part of the vector potential will be different. This arises due to the fact that for the form of the vector potential in (28), the resulting electric and magnetic fields will not satisfy the Maxwell-Ampère equation, $\nabla \times \mathbf{B}=\partial_{t} \mathbf{E}$, for finite time intervals. As an example, for a sinusoidal time dependence like $B(t)=B_{0} e^{i \omega t}$, the vector potential will be given by Bessel functions $[5,20]$

$$
\begin{aligned}
\mathbf{A}_{\text {in }} & =A_{1} J_{1}(\omega \rho) e^{i \omega t} \hat{\boldsymbol{\varphi}} \quad \text { for } \rho<\mathrm{R} \\
\mathbf{A}_{\text {out }} & =\left[C_{1} J_{1}(\omega \rho)+D_{1} Y_{1}(\omega \rho)\right] e^{i \omega t} \hat{\boldsymbol{\varphi}} \quad \text { for } \rho \geq \mathrm{R},
\end{aligned}
$$

where $A_{1}, C_{1}, D_{1}$ are constants and $J_{1}(x), Y_{1}(x)$ are Bessel functions of order 1 . For this more complex form for the vector potential, one can still calculate the magnetic and electric fields as in (29) and (30); of course now $\mathbf{B}_{\text {out }} \neq 0$. However even with the new form of the vector potential given in (33), one can still make the same arguments in (31) and (32) which led to the cancellation of the timedependent electric and magnetic contributions of the phase shift (see Ref. [5]). Thus one is left with only the static, time-independent contribution to the phase shift, given by the term $\frac{B_{0} R^{2} \Delta \varphi}{2}$ in (32). If one evaluates the phase shift from the vector potential for the infinitesimal path in Fig. 3 at time $t$, one obtains $\int \mathbf{A}_{\text {out }} \cdot d \mathbf{x} \rightarrow \frac{B_{0} R^{2} \Delta \varphi}{2}$, which then matches the result in (32).

Comparing the above calculations and discussion with the previous non-Abelian results, we see that in both Abelian and non-Abelian theories there is a cancellation between the time-dependent electric and magnetic contributions to the Aharonov-Bohm phase shift. For the nonAbelian case we have only shown this cancellation for two specific, time-dependent solutions and for special contours and surfaces. Although we have not shown this cancellation for the non-Abelian fields in general, we nevertheless conjecture that this is a feature of more general timedependent non-Abelian field configurations.

\section{SUMMARY AND CONCLUSIONS}

In this paper we have investigated the Aharonov-Bohm effect for time-dependent non-Abelian fields. In contrast to 
the Abelian Aharonov-Bohm effect, much less work has been done on even the time-independent non-Abelian Aharonov-Bohm effect-Ref. [3] is one of the few works to deal with the time-independent non-Abelian AharonovBohm effect. The reason for this most likely lies in the difficulty in experimentally setting up and controlling nonAbelian field configurations. In comparison, Abelian fields can be much more easily manipulated, e.g. setting up the magnetic flux tube, which is used in the canonical Abelian, Aharonov-Bohm setup. In this work, we studied (for the first time as far as we could determine from the literature) the Aharonov-Bohm effect for time-dependent nonAbelian fields. We did this using two specific, known time-dependent solutions (the Coleman plane wave solutions [6] and the time-dependent Wu-Yang monopole of [7]) and using specific contours and associated areas (see Figs. 1 and 2). There were two common results of this investigation: (i) The non-Abelian Aharonov-Bohm phase calculated via the fields [i.e. $\int F_{\mu \nu}^{a} T^{a} d \sigma^{\mu \nu}$ see (10)] did not agree in general with the Aharonov-Bohm phase calculated via the potentials [i.e. $\oint A_{\mu}^{a} T^{a} d x^{\mu}$ see (7)]. This point was already remarked upon by Coleman [6] as an example of the Wu-Yang ambiguity [8] for non-Abelian fields. (ii) There was a cancellation of the time-dependent contribution to the Aharonov-Bohm phase shift coming from the electric and magnetic non-Abelian fields. For the Coleman $(+)$ solution, this cancellation is given in Eq. (11) and for the time-dependent Wu-Yang monopole, this cancellation is given in Eqs. (19), (23) and (24).

In Sec. IV, we carried out a review of the time-dependent Abelian Aharonov-Bohm effect and made a comparison to the results from the time-dependent non-Abelian AharonovBohm effect from Secs. I-III. For both Abelian and nonAbelian fields, we found a cancellation of the time-dependent part of the phase shift. For the Abelian case, this was shown to occur generally_-see Eqs. (31) and (32). This led to the conjecture that this cancellation was also a feature of more general time-dependent non-Abelian fields and more general contours/area.

\section{ACKNOWLEDGMENTS}

M. B. was supported through a CSU Fresno College of Science and Mathematics FSSR grant and a Norma T. Craig grant.
[1] Y Aharonov and D. Bohm, Phys. Rev. 115, 485 (1959).

[2] W. Ehrenberg and R. E. Siday, Proc. Phys. Soc. London Sect. B 62, 8 (1949).

[3] P. A. Horváthy, Phys. Rev. D 33, 407 (1986).

[4] D. Singleton and E. Vagenas, Phys. Lett. B 723, 241 (2013).

[5] J. MacDougall and D. Singleton, J. Math. Phys. (N.Y.) 55, 042101 (2014).

[6] S. Coleman, Phys. Lett. 70B, 59 (1977).

[7] H. Arodź, Phys. Rev. D 27, 1903 (1983).

[8] T. T. Wu and C. N. Yang, Phys. Rev. D 12, 3843 (1975).

[9] K. Wilson, Phys. Rev. D 10, 2445 (1974).

[10] M. E. Peskin and D. V. Schroeder, An Introduction to Quantum Field Theory (Westview Press, Boulder, CO, 1995).

[11] B. Broda, Modern Nonlinear Optics, 2nd ed. edited by M. W. Evans (Wiley, New York, 2001), pp. 429-468.
[12] R. G. Chambers, Phys. Rev. Lett. 5, 3 (1960).

[13] A. Tonomura, N. Osakabe, T. Matsuda, T. Kawasaki, J. Endo, S. Yano, and H. Yamada, Phys. Rev. Lett. 56, 792 (1986).

[14] A. Noguchi, Y. Shikano, K. Toyoda, and S. Urabe, Nat. Commun. 5, 3868 (2014).

[15] B. Lee, E. Yin, T. K. Gustafson, and R. Chiao, Phys. Rev. A 45, 4319 (1992).

[16] N. G. van Kampen, Phys. Lett. 106A, 5 (1984).

[17] Yu. V. Chentsov, Yu. M. Voronin, I. P. Demenchonok, and A. N. Ageev, Opt. Zh. 8, 55 (1996).

[18] A. N. Ageev, S. Yu. Davydov, and A. G. Chirkov, Tech. Phys. Lett. 26, 392 (2000).

[19] L. Marton, J. A. Simpson, and J. A. Suddeth, Rev. Sci. Instrum. 25, 1099 (1954).

[20] B. Gaveau, A. M. Nounou, and L. S. Schulman, Found. Phys. 41, 1462 (2011). 